\title{
Effects of adults aging on word encoding in reading Chinese: evidence from disappearing text
}

\author{
Zhifang L Liu Corresp., ${ }^{1}$, Yun P Pan ${ }^{2}$, Wen T Tong ${ }^{3}$, Nina Liu ${ }^{4}$ \\ 1 Department of Psychology, Ningbo University, Ningbo, Zhejiang, China \\ 2 Department of Psychology, Guizhou Normal University, Guiyang, Guizhou, China \\ 3 Department of Psychology, Shanxi Normal University, Linfen, Shanxi, China \\ 4 Academy of Psychology and Behavior, Tianjin Normal University, Tianjin, Tianjin, China \\ Corresponding Author: Zhifang L Liu \\ Email address: Izhf2008@163.com
}

The effect of aging on the process of word encoding for fixated words and words presented to the right of the fixation point during reading of sentences in Chinese was investiga ted with two disappearing text experiments. The results of Experiment 1 showed that only the 40-ms onset disappearance of word $n$ disrupted young adults' reading performance. However, for old readers, the disappearance of word $n$ caused disruptions until the onset time was 120 ms. The results of Experiment 2 showed that the disappearance of word $n+1$ did not cause disruptions to young adults, but these conditions made old readers spend more time reading a sentence compared to the normal display condition. These results indicated a reliable aging effect on the process of word encoding when reading Chinese, and that the encoding process in the preview frame was more susceptible to normal aging compared to that in the fixation frame. We propose that sensory, cognitive, and specific factors related to the Chinese language are important contributors to these age-related differences. 
1 Zhifang Liu ${ }^{1}$, Yun Pan ${ }^{2}$, Wen Tong 3 , Nina Liu ${ }^{4}$

$2{ }^{1}$ Department of Psychology, Ningbo University, Ningbo, China

3 2Department of Psychology, Guizhou Normal University, Guiyang, China

$4 \quad{ }^{3}$ Department of Psychology, Shanxi Normal University, Linfen, China

$5 \quad{ }^{4}$ Academy of Psychology and Behavior, Tianjin Normal University, Tianjin, China

6 Corresponding author: Zhifang Liu, email: 1zhf2008@163.com 


\section{Effects of adults aging on word encoding in reading Chinese:}

\section{9 evidence from disappearing text}

Abstract: The effect of aging on the process of word encoding for fixated words and words presented to the right of the fixation point during reading of sentences in Chinese was investigated with two disappearing text experiments. The results of Experiment 1 showed that only the 40-ms onset disappearance of word $\mathrm{n}$ disrupted young adults' reading performance. However, for old readers, the disappearance of word $n$ caused disruptions until the onset time was $120 \mathrm{~ms}$. The results of Experiment 2 showed that the disappearance of word $\mathrm{n}+1$ did not cause disruptions to young adults, but these conditions made old readers spend more time reading a sentence compared to the normal display condition. These results indicated a reliable aging effect on the process of word encoding when reading Chinese, and that the encoding process in the preview frame was more susceptible to normal aging compared to that in the fixation frame. We propose that sensory, cognitive, and specific factors related to the Chinese language are important contributors to these age-related differences.

\section{INTRODUCTION}

Reading is an important practiced skill for daily life. This skill is acquired in early life and remains remarkably stable in adult age. However, many studies have recently revealed some subtle age differences in reading, that is, old adults spend more time to comprehend texts, make longer fixations, and more regressions than young adults (Kemper, Crow \& Kemtes, 2004; Kliegl, Grabner, Rolfs, Engbert, 2004; Stine-Morrow, Miller, \& Hertzog, 2006; Rayner, Reichle, Stroud, Williams, Pollatsek, 2006; Laubrock, Kliegl, \& Engbert, 2006; Rayner, Castelhano, \& Yang, 2009). Changes in optics and neural transmission that occur with normal aging often lead old adults to experience a range of subtle visual deficits (Owsley, 2011), which may contribute substantially to their decline in reading ability. Rayner et al. $(2006,2009)$ also proposed that these age-related differences are largely attributed to the sensory and cognitive decline associated with normal aging. However, the nature of this decline has yet to be fully determined. Of particular importance for the present study is that the aging effect on visual word encoding may be related to both the changes in visual sensory abilities and the cognitive decline associated with normal aging. Investigation of how visual word encoding changes with age will inform future developments of computational models for eye movement control, such as the E-Z Reader and SWIFT models, which have been shown to successfully simulate some of the aging differences in word processing for alphabetic languages (Laubrock et al., 2006; Rayner et al., 2006; Reichle, Rayner, \& Pollatsek, 2003; Engbert, Nuthmann, Richter, \& Kliegl, 2005). However, there are no models that have attempted to simulate aging changes when encoding Chinese words during reading.

The disappearing text paradigm, in which the fixated word $n$ or word $n+1$ disappears after a 
certain fixation duration (i.e., 40, 60, or $80 \mathrm{~ms}$ ), has been the most effective method to resolve the issue of visual word encoding. With this paradigm, researchers have found that most of the visual information of the fixated word necessary for reading can be acquired within the initial 50-60 ms during a fixation for young readers of English, which means that they are able to encode all the needed visual information of a fixated word within this time frame (Rayner, Inhoff, Morrison, Slowiaczek, \& Bertera, 1981; Liversedge et al., 2004; Blythe, Liversedge, Joseph, White, \& Rayner. 2009; Blythe, Häikiö, Bertam, Liversedge, \& Hyönä, 2011). Maybe the most striking result was that even when the word disappeared after a fixation of $60 \mathrm{~ms}$, a robust frequency effect on the target words remained, which meant that word processing influenced eye movements in reading, which is consistent with the cognitive-control models of eye movements (Rayner, Liversedge, White, \& Vergilino-Perez, 2003). Rayner, Liversedge, and White (2006) also investigated the visual encoding of word $n+1$ with disappearance experiments and found that the disappearance of word $n+1$, after word $n$ was fixated for $60 \mathrm{~ms}$, greatly impaired reading, which indicated not only the importance of visual coding for word $n+1$, but also that the time needed for encoding words in parafoveal vision is longer than for words in foveal vision when reading English. It should be noted that the E-Z Reader model can account not only for young adults' eye movement data for word $\mathrm{n}$ disappearing text but also for their performance in word $\mathrm{n}+1$ disappearing conditions during reading of English (Pollatsek, Reichle, \& Rayner, 2006; Reichle, Liversedge, Pollatsek, \& Rayner, 2009).

Clinical impairments are commonly found in the population of old adults, and these impairments have profound effects on reading performance (Paterson, McGowan, \& Jordan, 2013). Further evidence has revealed that reading performance decreases significantly with age even in people whose visual acuity is good (Lott, Schneck, Haegerstrom-Portnoy, Brabyn, \& Gildengorin, 2001). Moreover, given the nature of sensory and cognitive decline in old adults, it is likely that aging affects encoding of visual word information. Thus, it is safe to hypothesize that old adults need more time to encode words during reading. However, previous research on this topic in reading English has revealed that for old readers, the disappearance of the fixated word caused relatively greater reading slowdown compared to young adults, even in the 60-ms onset disappearing conditions. Due to the absence of an interaction between age group and disappearing onset when the control condition was removed, and the word frequency effect for both groups in the disappearing conditions, the authors argued that the effect of disappearing onset is comparable in old and young readers of English text (Rayner, Yang, Castelhano, \& Liversedge, 2010). Thus, these results indicated no age effects on visual word encoding when reading English. However, it has been an open question whether aging effects on visual word encoding are a language-specific phenomenon or whether they are universal and apply to other languages such as Chinese. Furthermore, it is important to explore the aging pattern of word encoding in parafoveal vision, as preview processing of words is common for both young and adult readers (Rayner et al., 2009; Rayner, Castelhano, \& Yang, 2010). However, research on this topic is still rare both in reading English and Chinese.

Encoding words of alphabetic languages during reading involves the discrimination of one- 
84 dimensional, linear combinations of letters or phonological units and then encapsulate them into

85

86

87

more permanent representations (Reichle et al., 2009). However, encoding Chinese words involves the recognition of the two-dimensional, pattern-like structure of characters, which engages unique mental processes (Zhang et al., 2012). Thus, comparing aging effects on visual word encoding of Chinese to those of English is extremely valuable for developing computational models that simulate aging differences during reading. As mentioned above, with the disappearing text paradigm, researchers have extensively investigated the time needed for encapsulating words in foveal and parafoveal vision (Rayner et al., 1981, 2003, 2006; Liversedge et al., 2004) and the developmental and aging issues of encapsulating the visual information of a fixated word (Blythe et al., 2009, 2011; Rayner et al., 2010). As previous studies have revealed, aging does not have an effect on encoding visual word information in the fovea during reading English (Rayner et al., 2010). In contrast to the well-documented effects of disappearing text and the effects of aging on reading English, however, empirical studies concerning disappearing text in reading Chinese have been relatively sparse and are still in their infancy. Only one study using the disappearing text paradigm was found - in which visual information of the fixated word necessary for sentence comprehension can be acquired within the initial 50-60 ms for young adult readers of Chinese - which indicated that they could encapsulate the fixated visual word information as quickly as young adult readers of English (Liu, Zhang, \& Zhao, 2011). Although researchers have investigated whether word difficulty factors (e.g., usage and visual complexity) affected young and old adults differently (Wang et al., 2016; Zang et al., 2016), there has been no study, as far as we know, that manipulated the exposure time of words to directly examine the aging effect on Chinese visual word encoding in reading.

In view of the substantial differences between the two script types, findings on word encoding of alphabetic scripts (such as English) during reading cannot be directly extended to reading Chinese. Firstly, Chinese writing is logographic whose written style is completely different from that of alphabetic text. Evidence has shown that the processes underlying lexical identification of Chinese words are very different from those of alphabetic languages (Zhou \& Marslen-Wilson, 2000). The prevalent assumption has been that Chinese lexical identification is a form-tomeaning process with little involvement of phonology; therefore, it appears that orthography dominates over phonology, and that orthographic encoding is a core process in Chinese word identification (Perfetti, Liu, \& Tan, 2005). Zhang et al. (2012) also posited that Chinese is a more thoroughly visual language compared to alphabetic scripts, and thus emphasizes the role of visual processing in word recognition. Secondly, Chinese is a language with no spaces to separate words in a text, and texts written in Chinese are formed by strings of box-like symbols (i.e., characters). Although series of studies conducted by Bai and colleagues have suggested the importance of words (opposed to characters) for reading and learning Chinese, which are the same as for alphabetic languages (Bai, Yan, Liversedge, Zang, \& Rayner, 2008; Blythe et al., 2012; Shen et al., 2012; Bai et al., 2013), character processing is the necessary stage for multicharacter word recognition (Li, Rayner, \& Cave, 2009; Shen, \& Li, 2012; Li, Bicknell, Liu, Wei, \& Rayner, 2014). Therefore, readers must segment character strings into words during preview (Yan, Kliegl, Shu, Pan, \& Zhou, 2010; Shu, Zhou, Yan, \& Kliegl, 2011; Yan, Zhou, Shu, \& 
125 Kliegl, 2015; Gu \& Li, 2015), which may lead to greater difficulty for old people when encoding

126

127

128

129

130

131

132

133

134

135

136

137

138

139

140

141

142

143

144

145

146

147

148

149

150

151

152

153

154

155

156

157

158

159

160

161

162

visual word information. With the characteristics of Chinese text mentioned above, aging effects on visual encoding of words during reading may be larger than those during reading English text. Thus, in this study, we employed two disappearing text experiments with longer interval onset times compared to previous research conducted by Rayner et al. (2010) to explore the effect of aging on the time needed for encoding words both in foveal and parafoveal vision (i.e., word $n$ and $n+1$ ) during reading of Chinese text.

The primary goal of the current study, therefore, was to examine age-related changes in visual word encoding. If such changes could be established, how much time would old adults need to encode the visual word information in foveal and parafoveal vision as well as young adult readers of Chinese? We further examined aging effects on the time needed for encoding not only the fixated word (word $n$ ) but also the word to the right of fixation (word $n+1$ ) when reading Chinese by two disappearing text experiments, respectively. The first experiment was conducted to explore aging effects on encoding word $n$, in which the onset times of the word $n$ disappearance were manipulated. In the second experiment, the same onset times of word $n+1$ disappearance were manipulated, and by doing so, we intended to clarify the aging effects on encoding word $n+1$ and compare it to the aging effects on encoding word $n$. The onset times of the disappearance manipulation in both experiments were 40,80,120, and $160 \mathrm{~ms}$, with a 40-ms interval of the disappearing manipulation. The logic of both experiments was straightforward. If the onset time were not sufficient for encoding before the word disappeared, normal reading performance would be impaired under this onset time of disappearing text. Moreover, if young and old adults had different requirements on the time needed for encoding the words to read normally, these differences should be revealed by the effectiveness of each onset times of disappearing manipulation to sustain normal reading performance in each age group.

\section{GENERAL METHOD}

\section{Human Ethics}

The data were anonymously analyzed. The subjects provided verbal and written informed consent by signing a form to receive money for their participation. The current study was approved by the ethics committee of the Department of Psychology of Ningbo University (approval number: 20150901).

\section{Participants}

Sixty adults from the University of Ningbo and local community participated in the experiments. Of these, 15 young adults $(M=20.5$ years, range $18-22$ years) and 15 old adults $(M$ $=66.7$ years, range 60-73 years) participated in Experiment 1 , and another 15 young adults $(M=$ 20.4 years, range 18-22 years) and 15 old adults $(M=66.8$ years, range 60-73 years) participated in Experiment 2. The young and old adult groups did not differ in number of years of schooling (15.8 years for the young adults and 15.4 years for the old readers). All participants were right-handed with normal or corrected-to-normal vision. Subjects with eyesight problems 
163

164

165

166

167

168

169

170

171

172

173

174

175

176

177

178

179

180

181

182

183

184

185

186

187

188

189

190

191

192

193

194

195

196

197

198

199

200

were asked to wear their glasses before they participated in the experiment, and there was no group difference in corrected vision scores by the Tumbling E acuity chart (old adults: $M=4.99$, $S E=0.15$; young adults: $M=4.97, S E=0.13 ; t=1.117, p>0.05)$. All participants reported that they could clearly see the text in the no disappearing condition after the practice phase in the experiment. They were all native Chinese speakers and were paid $¥ 30$ for participation. None of them was aware of the purpose of the experiment or had previously participated in other similar experiments.

\section{Apparatus}

The participants' movements of the right eye were recorded with an Eye Link 1000 device manufactured by SR Research Ltd. The eye tracker is an infrared video-based tracking system, and its camera samples the pupil location and pupil size at a rate of $1000 \mathrm{~Hz}$. This system also has high spatial resolution $\left(<0.01^{\circ} \mathrm{RMS}\right)$. The sentence stimuli were presented on a 19-inch DELL LCD monitor with a $1024 \times 768$ pixel resolution and refresh rate of $75 \mathrm{~Hz}$. The sentences were displayed in Song font, and the size of each Chinese character was $28 \times 28$ pixels subtending approximately $0.63^{\circ}$ visual angle. The distance between the participant and screen was $75 \mathrm{~cm}$.

\section{Design and Stimuli}

Both experiments followed a 2 (group: young adults $v s$. old adults) $\times 5$ (disappearing onset: no disappearing, $40 \mathrm{~ms}, 80 \mathrm{~ms}, 120 \mathrm{~ms}, 160 \mathrm{~ms}$ ) mixed design. The former variable was a betweensubjects factor and the latter a within-subjects factor. Experiment 1 explored whether disappearing word $\mathrm{n}$ influenced young and old Chinese readers' reading behavior, and Experiment 2 explored whether disappearing word $n+1$ influenced young and old Chinese readers' reading behavior. Both groups of participants read the Chinese sentences in a normal condition (control) and four experimental conditions in which the word $n$ or word $n+1$ disappeared after the designated interval (40, 80, 120, or $160 \mathrm{~ms}$ ). An immediate re-fixation on word $\mathrm{n}$ did not result in its re-appearance until the reader made an eye movement to a new word in the disappearing manipulations. Fig. 1 shows an example of the disappearing manipulations in detail; when the reader fixated on word “垃圾," which was word n, it disappeared after 40, 80, 120 , or $160 \mathrm{~ms}$ and was not presented until the reader made an eye movement to a new word. When the reader made an eye movement and fixated on word “通道," the disappeared word “垃

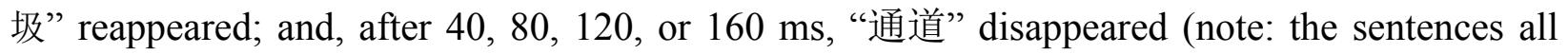
contained 7 or 8 two-character words).

\section{Figure 1 insert here}

Fig. 2 shows an example of the disappearing manipulations in detail; when the reader fixated on word “垃圾," which was word n, the word “通道," which was word n+1, disappeared after 40, 80,120 , or $160 \mathrm{~ms}$ and was not presented again until the reader made an eye movement to a new word. When the reader made an eye movement and fixated on word “通道," the disappeared word re-appeared, and after 40, 80, 120, or 160 ms, “需要” disappeared. 
It is important to note that the refresh rate of the screen can affect the precise timing of a display. The disappearing text manipulations were initiated when the eye fixated on word $\mathrm{n}$. The refresh rate of the screen was $75 \mathrm{~Hz}$ in the present study, therefore, in each disappearing condition, there was a potential additional $15 \mathrm{~ms}$ of delay before the words disappeared after the eye moved to word n. Thus, the actual onset times of the disappearing manipulations in both experiments (Experiments 1 and 2) were 55, 95, 135, or $175 \mathrm{~ms}$.

Eighty sentences were used as stimuli in the experiments. These sentences all contained 7 or 8 two-character words. Considering that $72 \%$ of Chinese words are two-character words-when word tokens are taken into account, $27 \%$ words are two-character words - it was easy to make up fluent Chinese sentences with only two-character words (Li, Gu, Liu, \& Rayner, 2012). Ten college students, who did not participate in the experiments, were asked to rate the difficulty of difficulty score was 1.65 . Another 10 college students, who did not participate in the experiments, were asked to rate the naturalness of these sentences on a 7-point scale (e.g., a score of 7 was "very natural"). The resulting mean naturalness score was 6.30. Each sentence was shown in one of five display conditions. All eighty sentences were randomized and sampled using a Latin square, so that each participant saw sixteen sentences shown in each of the five display conditions: a control and four disappearing display conditions. By doing this, we wanted to ensure that all sentences were shown equally often in the five display conditions and prevent repetition of any sentence for each participant. Sentences were shown to each participant in a randomized order across two sessions.

There were 12 additional sentences (five of which had questions) for practice in the first session. The second session was the formal experiment. To confirm that participants were reading the sentences carefully, there were 27 filler sentences that were randomly inserted throughout the block. A Yes/No comprehension question was presented at the end of each filler sentence, and the participants were asked to answer the questions by manually pressing the buttons.

\section{Procedure}

Participants were tested individually. Before the experiment, they were informed that they would read sentences presented in different disappearing manners. The participants were instructed to read and understand the sentences and were asked to push a button box to terminate the current display when a sentence was completed. A comprehension task occasionally appeared after a sentence, and the participants were asked to answer a Yes/No question by pressing different buttons. A chin rest was inserted to ensure that the participants' heads remained still. A calibration procedure was executed prior to the beginning of the experiment, in which the participant was instructed to look at each of three fixation points arranged along a 
238 horizontal line across the center of the screen. Once the eye tracker had been calibrated with satisfactory accuracy (mean error less than $0.5^{\circ}$ ), the sentence was presented. The eye tracker was re-calibrated before the next trial when the error from drift correction of the current trial was greater than $0.5^{\circ}$. There were 4-6 re-calibrations for each subject. The experiment was approximately 25 minutes in length.

\section{Data Analysis}

Since the purpose of this study was to investigate aging effects on visual word encoding by checking the differences in time needed for word encoding between young and old adults, we compared each disappearing condition to the control separately in young and old adults. One word-independent measure, reading time, was the most important reference measure with the disappearing text paradigm (Blythe et al., 2009); three word-dependent measures, such as mean gaze duration (mean gaze duration on all the words in a sentence), probability of skipped words in the initial pass when reading a sentence, and probability of regressed words during reading were analyzed as supplementary data. The reason to adopt the reading time measure as the main reference dependent variable is that readers may trade off fixation duration and number during disappearing text reading (Rayner et al., 1981; Liversedge et al., 2004; Liu et al., 2011), which in turn could lead the word-dependent measures not being sensitive to the word encoding process. All eye movement data above or below three standard deviations from the mean were excluded; as a result, $3.7 \%$ of the data in total were removed prior to conducting the analyses. Data were analyzed by linear mixed models (LMM) using the lme4.0 package (Bates, Maechler, Bolker, \& Walker, 2016) and the latest version of R, which was retrieved from https://www.r-project.org/. The full models included random intercepts for participants and stimuli and included additional random slopes in which participants and stimuli were allowed to vary with respect to the fixed effects. If the model did not converge, we sequentially simplified the maximal model until it converged. The models were run on log-transformed reading time and mean gaze duration. Logistic LMM models were used for analyzing the probability of skipped words and probability of regressed words. The contrasts of the main effects were defined with sliding contrasts in the MASS package (Venables \& Ripley, 2002). The last version of this package was also retrieved from https://www.r-project.org/. We report regression coefficients $(b)$, standard errors $(S E)$, and $t$ values $(t=b / S E)$, regression coefficients approximately twice as large as their $S E$ (abs $[t$ or $z$ value] $>2$ ) are interpreted as significant at the $5 \%$ level.

The rationale of the disappearing text paradigm is to increase the amount of visual information available per fixation and to test the minimum amount of time for encoding the disappeared word to maintain a normal reading behavior when no viewing restriction is applied. The goal of the present study was also to investigate how young and old readers of Chinese differ in the time needed for encoding the visual word information into a more stable code. Therefore, we consider the treatment contrast between young and old groups to be particularly well suited for the goal of the present study. Inferential statistics were based on a prior treatment contrast with the no disappearing condition as the reference category for the four disappearing text conditions. Then, the group differences when reading the text without disappearing conditions from the two 
experiments were explored. Furthermore, older adults' reading data from the two experiments were compared to explore differences of aging between encoding word $n$ and word $n+1$. In this analysis, experiment (Experiment 1 vs. Experiment 2) was specified as a fixed effect, and there were four contrasts (contrast 1: no disappearing vs. 40-ms disappearing onset, contrast 2: no disappearing vs. 80 -ms disappearing onset, contrast 3: no disappearing vs. 120-ms disappearing onset, contrast 4: no disappearing vs. 160-ms disappearing onset). The model provided statistics with respect to five main effects (experiment, contrast 1, contrast 2, contrast 3, and contrast 4) and four interactions (experiment and contrast 1, experiment and contrast 2, experiment and contrast 3 , experiment and contrast 4$)$.

\section{EXPERIMENT 1}

Experiment 1 investigated the differences in time needed for encoding the visual information of the fixated word into a more stable code between young and old adults. We presented the text either entirely as normal or in the disappearing text mode, in which word $\mathrm{n}$ disappeared after the designated interval $(40,80,120$, or $160 \mathrm{~ms})$ from the time it was fixated.

\section{Results}

The mean accuracy of all participants for the 27 comprehension sentences exceeded $80 \%$, and there were no significant effects of display condition and age group $(p s>0.10)$. Namely, the comprehension levels were high in all situations, which indicated that participants read and fully understood the sentences. All the data are summarized in Table 1.

\section{Table 1 insert here}

Reading time. Only the 40-ms disappearing onset condition prolonged young adults' sentence reading time compared to the control, $b=0.040, S E=0.015, t=2.623$; while the other disappearing manipulations did not interrupt their normal reading performance, $b s<0.022$, SEs $>0.012$, $t s<1.60$. However, in old adults, the 40-ms and 80-ms disappearing onset conditions differed from the control (40-ms onset vs. control: $b=0.062, S E=0.019, t=3.235,80$-ms onset vs. control, $b=0.041, S E=0.015, t=2.797$ ), with the shorter disappearing onset producing longer reading times. The 120 -ms and $160-\mathrm{ms}$ onset disappearing conditions did not interrupt older adults' reading performance, $b s<0.019, S E s>0.013$, $t s<0.35$.

Mean gaze duration. The disappearing manipulations influenced young and old adults differently. All the disappearing onset conditions prolonged the young adults' gaze duration compared to the no disappearing condition, $b s>0.018$, SEs $<0.01$, $t s>2.12$; with longer onset times, the mean gaze duration was shorter. However, in old adults, the 40-ms disappearing onset condition led to shorter gaze duration compared to the no disappearing condition, $b=-0.031, S E$ $=0.015, t=-2.110$. There were no differences between the other disappearing conditions and the control, abs $[b$ values $]<0.011, S E s>0.008$, abs $[t$ values $]<0.992$, that is, the longer disappearing onsets did not affect mean gaze duration. 
314 Probability of skipped words. The word $\mathrm{n}$ disappearing manipulations affected the skip 315 probability of young and old adults differently. All disappearing conditions did not differ from 316 the no disappearing condition for young adults, $b s<0.036, S E s>0.097, Z s<0.97$; however, the 317 40-ms and 160-ms disappearing conditions led old adults to skip words more often compared to 318 the no disappearing condition, $b s>0.285$, SEs $<0.133, Z s>2.195$.

319 Probability of regressed words. Compared to the control, none of the disappearing conditions influenced the regression probability of words in young adults, abs $[b$ values $]<0.07$, $S E s>0.10$, abs $[Z$ values $]<0.571$. However, the $40-\mathrm{ms}, 80-\mathrm{ms}$, and $160-\mathrm{ms}$ disappearing conditions made old adults regress more often than the control condition, $b s>0.245$, SEs $<$ $0.156, Z s>2.059$, with shorter disappearing onset increasing the probability of regressed words.

\section{Discussion}

The most important aspect of the sentence reading time data is that old adults were much more disrupted by the disappearance of the fixated word than were the young adults, and that the more immediate disappearing onset was more disruptive to text processing. In particular, we found that the disappearance of word $\mathrm{n}$ influenced reading in the young and aged groups differently, that is, only the 40-ms onset of the fixated word disappearing condition interrupted the reading time in the young adults, which suggested that the time needed for young Chinese readers to encode the visual information of word $\mathrm{n}$ for normal reading is about 55-95 ms. Thus, the results from this experiment replicated prior findings both in English and Chinese (Rayner et al., 1981; Liversedge et al., 2004; Liu et al., 2011). However, for old adults, the disappearance of word $n$ prolonged the reading time until the disappearing onset was $135 \mathrm{~ms}$ (120 ms disappearing onset). That is, the period needed for visual encoding of the fixated word was 95-135 ms for old adults, which is inconsistent with findings on reading English, in which the authors concluded that the disappearing onset effect was comparable in old and young English readers (Rayner et al., 2010).

The word-dependent eye movement measures provided additional evidence for the effects of normal aging on the time needed for visual encoding of fixated words. In particular, young and old adults adopted a completely different oculomotor strategy to read disappearing text. As seen from Table 1 and Figure 3, young adults gazed at word of interests longer, skipped more words, and made slightly more regressions during disappearing text reading. Although the disappearing onset effects on the probability of skipped and regressed words were not reliable, the higher skip probability traded off the longer mean gaze duration, which in turn blunted the negative influence of the disappearing manipulations on sentence reading time. In contrast, the disappearing manipulations led old adults to gaze at words for shorter times and make more skips in the first pass of reading; the disappearing manipulation also made old people regress more often compared to the no disappearing condition. It is easy to understand that more regressions caused a longer reading time. The differences between old and young adults manifested when reading in the word $\mathrm{n}$ disappearing conditions. Based on the reading time data, 
352

353

354

355

356

357

358

359

360

361

362

363

364

365

366

367

368

369

370

371

372

373

374

375

376

377

378

379

380

381

382

383

384

385

386

these results suggested that it was insufficient for old adults to visually encode the fixated word within the 95-ms display of visual information (i.e., 80-ms onset disappearance of word $\mathrm{n}$ still disrupted old adults' reading performance), so that they had to comprehend the text by recognizing the word on the right side of fixation (i.e., word $n+1$ ). These findings confirmed that compared to the young adults, old readers need more time to encode the visual information of the fixated word.

\section{EXPERIMENT 2}

Experiment 2 investigated the differences in time needed for encoding the visual information of word $n+1$ for normal reading performance between young and old adults. We either presented the text entirely as normal or presented it in the disappearing paradigm in which word $n+1$ disappeared after the designated interval $(40,80,120$, or $160 \mathrm{~ms})$ from when word $\mathrm{n}$ was fixated.

\section{Results}

The mean accuracy of all participants for the comprehension sentences exceeded $80 \%$. There were no reliable effects for the display conditions and age groups ( $p s>0.10)$. All eye movement data above or below three standard deviations from the mean were excluded, and $2.9 \%$ of the data in total were removed prior to conducting the analyses. The dependent measures are summarized in Table 2.

\section{Table 2 insert here}

Reading time. Similar to Experiment 1, we contrasted all the disappearing conditions with the no disappearing condition for young and old adults separately, and found that the word $n+1$ disappearing manipulations affected young and old readers differently. That is, compared to the no disappearing condition, none of the disappearing manipulations interrupted the young adults' normal reading performance, $b s<0.023$, SEs $>0.014$, $t s<1.41$. However, all the disappearing manipulations interrupted the old readers' normal reading performance, $b s>0.092, S E s<0.023$, $t s>4.516$, with shorter disappearing onset producing longer reading times.

Mean gaze duration. Compared to the no disappearing condition, all the disappearing conditions prolonged the young adults' gaze duration on the word of interest, $b s>0.024$, SEs $<$ 0.015 , $t s>2.265$. The $80-\mathrm{ms}, 120-\mathrm{ms}$, and $160-\mathrm{ms}$ disappearing conditions also prolonged the old adults' gaze duration, $b s>0.053$, SEs $<0.020$, $t s>3.115$, but the 40-ms disappearing condition did not influence old adults' mean gaze duration, $b=0.041, S E=0.023, t=1.73$.

Probability of skipped words. All the disappearing conditions increased the young adults' skip probability, $b s>0.244, S E s<0.089, Z s>2.788$. In old adults, all the disappearing conditions increased skip probability compared to the control, $b s>0.628$, SEs $<0.181, Z s>3.65$, with the shorter disappearing onset producing higher skip probability.

Probability of regressed words. Compared to the control, none of the disappearing 
387 conditions influenced the word regression probability in young adults, $b s<0.163$, SES $>0.126$, $388 Z s<1.467$. However, all the word $\mathrm{n}+1$ disappearing manipulations caused old adults to regress 389 more often, $b s>0.618$, SEs $<0.165, Z s>3.961$, with the shorter disappearing onset producing 390 more regressions.

\section{Discussion}

392 The aging effect on the process of encoding word $n+1$ when reading Chinese was explored in 393 this experiment. It was again confirmed that old adults were much more disrupted by the 394 disappearance manipulations than were the young adults, and that the more immediate 395 disappearing onset was more disruptive to processing. As described in the previous section, none 396 of the word $n+1$ disappearance manipulations impaired the normal reading performance of young 397 adults. However, for the old adults, the disappearing manipulations prolonged sentence reading 398 time, with the shortest disappearing onset time (40 ms) causing the greatest interruptions. The 399 results of the young adults are inconsistent with previous studies in English, which indicated that 400 the 60-ms onset time disappearance of word $n+1$ impaired young readers' normal reading 401 performance (Rayner et al., 2006). The results on old adults indicated that it becomes more 402 difficult to encode word $\mathrm{n}+1$ within the limited time, and even $175 \mathrm{~ms}$ might not be sufficient 403 (the 160-ms onset time of word $n+1$ disappearing condition still impaired the old adults' reading 404 performance). Thus, these data indicated that old adults' reading suffered more seriously when encoding word $n+1$ than when encoding the fixated word.

The word-dependent eye movement measures provided additional evidence to support the conclusions made from the reading time data. It was found that readers also adopted a strategy of trading off gaze duration, regressions, and skips to read the sentences treated by the word $n+1$ disappearing manipulations. As seen in Table 2, young adults adopted a totally different oculomotor strategy to read word $\mathrm{n}+1$ disappearing text compared to the old readers. In particular, the disappearance of word $n+1$ affected the mean gaze duration for young adults and affected the probability of skipped words almost equally for both young and old adults. The longer mean gaze duration during the word $n+1$ disappearing manipulations were traded off with a higher skip probability for both groups, that is, when reading word $n+1$ disappearing text, readers were inclined to fixate fewer words with longer reading time. It is quite likely that the trade-off between fixation number and time occurred similarly in this experiment, which led to longer gaze duration in the disappearing text reading for both groups; however, the reduced preview caused by the word $n+1$ disappearing text may have also contributed to the prolonged gaze duration of old readers. The measure of regressions further revealed the group differences. It is crucial to illustrate the differences between young and old adults in reading time and regressions, as seen from Table 2; none of the disappearance onsets of word $n+1$ brought on more regressions for the young adults but brought on more regression for old adults.

\section{3}

\section{COMPARISON BETWEEN EXPERIMENTS}

424 It should be pointed out that across the two experiments, the control conditions differed very 
425 little and any variability could be due to the between-participant manipulation, since different

426

subjects participated in the two experiments. In order to better understand the age-related differences in performance when reading Chinese, a series of analyses were conducted to compare the differences between young and old adults in the no-disappearing conditions from the two experiments. As evident from these analyses, when reading the text under normal display conditions, old adults took more time than young adults (young adults: $M=2502 \mathrm{~ms}$, old adults: $M=3513 \mathrm{~ms} ; b=0.186, S E=0.049, t=3.765$ ), had longer gaze duration (young adults: $M=$ $256 \mathrm{~ms}$, old adults: $M=331 \mathrm{~ms} ; b=0.185, S E=0.049, t=3.756)$, and tended to make more regressions than young adults (young adults: $M=0.24$, old adults: $M=0.32 ; b=0.551, S E=$ $0.221, t=2.489$ ), which is consistent with results from research on aging effects of alphabetic script reading (Kemper et al., 2004; Kliegl et al., 2004; Rayner et al., 2006, 2009). However, old Chinese readers skipped fewer words than young readers (young adults: $M=0.233$, old adults: $M$ $=0.107 ; b=-1.007, S E=0.213, t=-4.723$ ). This finding differs from aging effects on reading English, which suggested that compared to old readers of English (Rayner et al., 2009), old Chinese adults adopted a more cautious oculomotor strategy when reading Chinese, and they were more inclined to look at the words one-by-one during reading. These findings are valuable to understand the aging effects on word encoding when reading Chinese.

In order to better understand the different aging effects on word encoding between words in foveal and parafoveal locations, the old adults' reading data in both experiments were further compared. In this analysis, only the reading time was considered, because old adults adopted two completely different oculomotor strategies to read in the disappearing text conditions of Experiment 1 and 2 (as seen in Table 1 and 2, respectively), which may have caused the worddependent measures not being susceptible to the different aging effects on encoding word $\mathrm{n}$ and word $\mathrm{n}+1$. The results showed that old adults spent more time to read the sentences in Experiment 2 than to read the sentences in Experiment $1, b=0.113, S E=0.037, t=3.006$. All the contrasts were reliable, $b s>0.056, S E s<0.008$, $t s>7.224$, and so were the interactions, $b s>$ $0.07, S E s<0.02$, $t s>4.513$, which indicated that the disappearance of word $\mathrm{n}+1$ impaired old adults' reading performance more seriously than the disappearance of word $n$. This indicated that the aging effect on encoding word $n+1$ was larger than that on encoding word $n$.

\section{Figure 3 insert here}

\section{Figure 4 insert here}

\section{GENERAL DISCUSSION}

With two disappearing text experiments, the aging effect on visual word encoding (word $\mathrm{n}$ and $\mathrm{n}+1$ ) was investigated during reading Chinese. The present study provides evidence concerning the word encoding process of older Chinese readers. Generally, it was found that compared with young adults, old adults read more slowly, gaze at words for longer, and make more regressions (see Tables 1 and 2, Figures 3 and 4, and section on comparison between experiments). This 
462

463

464

465

466

467

adds to the growing evidence that old readers suffer from greater reading difficulties (Kemper et al., 2004; Kliegl et al., 2004; Stine-Morrow et al., 2006; Rayner et al., 2006, 2009; Laubrock et al., 2006). We also found some age changes that are specific of Chinese, that is, compared to reading alphabetic languages such as English and German, old adults reading Chinese employ a more cautious eye movement strategy (i.e., they skip words less frequently), which is consistent with previous studies (Liu, Liu, Han, \& Paterson, 2015; Wang et al., 2016; Zang et al., 2016). These researchers proposed that increased difficulty in processing word boundary in the preview phase with age causes older adult readers to use a more careful reading strategy to compensate for this difficulty. However, only a few studies have directly explored the aging effect on parafoveal processing of words. The disappearing text paradigm to investigate aging effects on processing of visual information of word $n$ and $n+1$ is particularly well suited to assess this issue.

Although previous studies have shown that young adult readers of Chinese need about the same time to encode the fixated word as readers of alphabetic languages, it does not mean that both languages share the same pattern of normal aging in word encoding during reading. The differences in time needed for encoding word $n$ between young and old adults during reading Chinese were investigated in Experiment 1. The results indicated that young adults can encode the visual information of a fixated word within 55-95 ms display time (i.e., 80-ms onset disappearance of word $\mathrm{n}$ did not disrupt young adults' reading performance), which is generally consistent with prior research both for reading English and Chinese, suggesting that young adults can read fairly well when they see the fixated word for about $55 \mathrm{~ms}$ before it disappears (Rayner et al., 2003; Liversedge et al., 2004). However, old Chinese adults need more time to encode the visual information of word n (i.e., 95-135 ms), which is not consistent with findings from old readers of English (Rayner et al., 2010). Comparing the results of this experiment to those obtained by Rayner and colleagues (Rayner et al., 2010), confirms that aging has a more serious impact on encoding fixated words when reading Chinese. We propose two possible factors for this difference. The first is that Chinese readers may rely more heavily on the preview process than English readers. The second is that word identification of Chinese text is a form-to-meaning process with more emphasis on visual processing (Zhou \& Marslen-Wilson, 2000; Perfetti et al., 2005; Zhang et al., 2012). Both factors may also interact with the visual decline (especially the disproportionate decline in peripheral vision); thus, making the encoding of visual word information during reading Chinese more susceptible to normal aging. The second experiment was conducted to check the first factor directly.

With the word $n+1$ disappearing text manipulations, we explored the aging effects on the process of encoding word $\mathrm{n}+1$ in Experiment 2. Rayner et al. (2006) observed that the 60-ms onset disappearance of word $n+1$ impaired young adults' reading performance of English text equally compared to its immediate disappearance. According to Pollatsek et al. (2006), attention plays a key role in encapsulating the visual information into a more permanent representation. By this account, $60 \mathrm{~ms}$ were sufficient for encoding the fixated word but not to identify the fixated word and then shift attention to word $n+1$ in time for it to be encoded. Thus, this is inconsistent with the attention gradient model, which assumes that multiple words are encoded in 
502

503

504

505

506

507

508

509

510

511

512

513

514

515

516

517

parallel because, if attention is allocated to both word $n$ and word $n+1$, there should be sufficient time to convert the visual information of both words into an orthographic code, enabling lexical processing of either word to continue without disruption after they disappear (Reichle et al., 2009). However, as seen from the results and discussion section for Experiment 2, none of the word $n+1$ disappearing conditions disrupted young Chinese adults' reading time (see Table 2 and Figure 3). Word skip probability was traded off by longer mean gaze duration in the disappearing manipulations compared with the control condition in young adults (see Table 2 and Figure 4). Thus, these results indicated that they could encode the visual information of word $n$ and $n+1$ into a relatively stable code in parallel. Given that young adults still needed some time to encode the visual word information within a fixation frame (as seen from Experiment 1), it is safe to conclude that young Chinese adult readers could encode the characters in word $n$ and $n+1$ successfully within $55 \mathrm{~ms}$ after they first fixated on word $\mathrm{n}$. (If the output of encoding word $\mathrm{n}+1$ is word-based, the reader should have no need to re-encode it when it is in the fixation frame.) Thus, the results of young adults when reading word $n+1$ disappearing text is consistent with the notion proposed by Li and his colleagues who argued that Chinese readers process characters in parallel during reading (Li et al., 2009; Ma, Li, \& Rayner, 2015).

The results of Experiment 2 suggest that young adult readers of Chinese are more inclined to encode the text in parallel than young adult readers of English, which also indicates that Chinese readers rely on the preview process. The influence of the word $n+1$ disappearing manipulations on old adults' reading is also informative for developing computational models of eye movement control when reading Chinese. All the word $n+1$ disappearing manipulations interrupted old adults' reading performance, in that they exhibited longer gaze duration and made more regressions when reading disappearing text than when reading no disappearing text (see Table 2 and Figure 4). Thus, both the word-independent and word-dependent variables revealed that 175 ms were not sufficient to encode word $\mathrm{n}+1$ for old readers. Comparing the old adults' reading time measure in Experiment 1 to that in Experiment 2 confirmed that the disappearing onset of word $n+1$ interrupted reading more seriously than the same disappearing onset of word $n$, which meant that old adults needed more time to encode word $n+1$ compared to word $n$. Old adults' reading suffered more when they encoded the text of word $n+1$ than when they encoded the fixated text, which confirmed that Chinese readers may rely more heavily on the preview process than English readers. Previous studies claimed that old people have difficulty segmenting words in the preview phase, which leads them to adopt a more careful reading strategy (Wang et al., 2016; Zang et al., 2016). Although the results of the present experiments do not refute this deduction, it can be concluded that the lower efficiency in encoding the characters of the previewed word was also an important contributor to the more cautious eye movement strategy adopted by old adults, which was a specific age change in reading Chinese.

The reasons why old Chinese readers had greater difficulty encoding the visual information of the previewed word than of the fixated word consisted of sensory, cognitive, and factors specific to Chinese; all of these factors are not mutually exclusive contributors to this difference. We propose that the sensory factor may be the most important contributor to this aging effect when 
542 reading words in the two locations. As a previous study has shown, visual acuity decreases with 543 normal aging disproportionately for peripheral vision relative to regions that are closer to the 544 fixation location (Cerella, 1985). Paterson, McGowan, and Jordan (2013) also found that old 545 adults gradually lose the ability to process detailed visual information in both foveal and 546 parafoveal regions and rely much more on coarse-scale components and a much wider region of 547 text when reading compared to young adults. Given that the perceptual span and preview word 548 segmentation when reading Chinese was mediated by visual factors (e.g., font size of characters),

\section{REFERENCE}

Bai X, Liang F, Blythe H I, Zang C, Yan G, Liversedge S P. 2013. Interword spacing effects on the acquisition of new vocabulary for readers of Chinese as a second language. Journal of Research in Reading 36: S4-S17 DOI 10.1111/j.14679817.2013.01554.x 
582

583

584

585

586

587

588

589

590

591

592

593

594

595

596

597

598

599

600

601

602

603

604

605

606

607

608

609

610

611

612

613

614

615

616

617

618

619

620

621

622

Bai X, Yan G, Liversedge S P, Zang C, Rayner, K. 2008. Reading spaced and unspaced Chinese text: Evidence from eye movements. Journal of Experimental Psychology: Human Perception and Performance 34: 1277-1287 DOI 10.1037/00961523.34.5.1277

Bates D, Maechler M, Bolker B, Walker S. 2016. Linear Mixed-Effects Models using 'Eigen’ and S4. R Package Version 1.1-12. Retrieved from $<$ https://cran.r-project.org/web/packages/>

Blythe H I, Liang F, Zang C, Wang J, Yan G, Bai X, \& Liversedge S P. 2012. Inserting spaces into Chinese text helps readers to learn new words: An eye movement study. Journal of Memory and Language 67: 241-254 DOI 10.1016/j.jml.2012.05.004

Blythe H I, Häikiö T, Bertam R, Liversedge S P, Hyönä J. 2011. Reading disappearing text: Why do children refixate words. Vision Research 51, 84-92 DOI 10.1016/j.visres.2010.10.003

Blythe H I, Liversedge S P, Joseph H S S L, White S J, Rayner K. 2009. Visual information capture during fixations in reading for children and adults. Vision Research 49: 1593-1591 DOI 10.1016/j.visres.2009.03.015

Cerella J. 1985. Age-related decline in extrafoveal letter perception. Journal of Gerontology 40: 727-736 DOI 10.1093/geronj/40.6.727

Engbert R, Nuthmann A, Richter E M, \& Kliegl R. 2005. SWIFT: A dynamical model of saccade generation during reading. Psychological Review, 112, 777-813 DOI 10.1037/0033-295X.112.4.777

Gu J, \& Li X. 2015.The effects of character transposition within and across words in Chinese reading. Attention, Perception, \& Psychophysics, 77, 272-281 DOI 10.3758/s13414-014-0749-5

Ishida T, Ikeda M. 1989. Temporal properties of information extraction in reading studied by a text-replacement technique. Journal of the Optical Society A: Optics and Image Science 6: 1624-1632 DOI 10.1364/JOSAA.6.001624

Kemper S, Crow A, Kemtes K. 2004. Eye-fixation patterns of high and low-span young and older adults: down the garden path and back. Psychology and Aging 19: 157-170 DOI 10.1037/0882-7974.19.1.157

Kliegl R, Grabner E, Rolfs M, Engbert R. 2004. Length, frequency, and predictability effects of words on eye movements in reading. European Journal of Cognitive Psychology 16: 262-284 DOI 10.1080/09541440340000213

Laubrock J, Kliegl R, Engbert R. 2006. SWIFT explorations of age differences in eye movements during reading. Neuroscience and Biobehavioral Reviews 30: 872-884 DOI 10.1016/j.neubiorev.2006.06.013

Li X, Bicknell K, Liu P, Wei W, Rayner K. 2014. Reading is fundamentally similar across disparate writing systems: A systematic characterization of how words and characters influence eye movements in Chinese reading. Journal of Experimental Psychology: General 143: 895-913 DOI 10.1037/a0033580.

Li X, Gu J, Liu P, \& Rayner K. 2012. The advantage of word-based processing in Chinese reading: evidence from eye movements. Journal of Experimental Psychology: Learning, Memory, and Cognition. 39, 879-889 DOI 10.1037/a0030337

Li X, Rayner K, Cave K P. 2009. On the segmentation of Chinese words during reading. Cognitive Psychology 58: 525-552 DOI 10.1016/j.cogpsych.2009.02.003

Liu P, Liu D, Han B, \& Paterson K B. 2015. Aging and the optimal viewing position effect in Chinese. Frontiers in Psychology, 6:1656 DOI 10.3389/fpsyg.2015.01656.

Liu Z, Zhang Z, \& Zhao Y. 2011. The Units Saccade Targeting Based on and Words Procession Style in Chinese Reading: Evidences from Disappearing Text. Acta Psychologica Sinica, 43: 608-618 DOI 10.3724/SP.J.1041.2011.00608.

Liversedge S P, Rayner K, White S J, Vergilino-Perez D, Findlay J M, Kentridge R W. 2004. Eye movements when reading disappearing text: is there a gap effect in reading? Vision Research 44: 1013-1024 DOI $\quad$ 10.1016/j.visres.2003.12.002

Lott L A, Schneck M E, Haegerstrǒm-Portnoy G, Brabyn J A, Gildengorin G L. 2001. Reading performance in older adults with good acuity. Optometry \& Vision Science, 78: 316-324, DOI 10.1097/00006324-200105000-00015

Ma G, Li X, \& Rayner K. 2015. Readers extract character frequency information from nonfixated-target word at long pretarget 
fixations during Chinese reading. Journal of Experimental Psychology: Human Perception and Performance, 41, 1409-1419 DOI $10.1037 / x h p 0000072$

Owsley C. 2011. Aging and vision. Vision Research, 51: 1610-1622 DOI 10.1016/j.visres.2010.10.020

Paterson K B, McGowan V A, Jordan T R. 2013. Effects of adult aging on reading filtered text: evidence from eye movements. PeerJ 1:e63 DOI 10.7717/peerj.63

Perfetti C A, Liu Y, Tan L H. 2005. The lexical constituency model: Some implications of research on Chinese for general theories of reading. Psychological Review 112: 43-59 DOI org/10.1037/0033-295X.112.1.43

Pollatsek A, Reichle E D, Rayner K. 2006. Test of the E-Z Reader mold: Exploring the interface between cognition and eye movement control. Cognitive Psychology 52: 1-56 DOI 10.1016/j.cogpsych.2005.06.001

Rayner K, Castelhano M S, Yang J. 2009. Eye movements and the perceptual span in older and younger readers. Psychology and Aging 24: 755-760 DOI 10.1037/a0014300

Rayner K, Castelhano M S, Yang J. 2010. Preview benefit during eye fixations in reading for older and younger readers. Psychology and Aging 25: 714-718 DOI 10.1037/a0019199

Rayner K, Inhoff A, Morrison R, Slowiaczek M L, Bertera J H. 1981. Masking of foveal and parafoveal vision during eye fixations in reading. Journal of Experimental Psychology: Human Perception and Performance 7: 167-179 DOI org/10.1037/0096-1523.7.1.167

Rayner K, Liversedge S P, White S J, Vergilino-Perez D. 2003. Reading disappearing text: Cognitive control of eye movements. Psychological Science 14: 385-388 DOI 10.1111/1467-9280.24483

Rayner K, Reichle E D, Stroud M J, Williams C C, Pollatsek A. 2006. The effects of word frequency, word predictability, and font difficulty on the eye movements of young and elderly readers. Psychology and Aging 21: 448-465 DOI 10.1037/08827974.21.3.448

Rayner K, Yang J, Castelhano M S, Liversedge S P. 2010. Eye movements of older and younger readers when reading disappearing text. Psychology and Aging, 26: 214-223 DOI 10.1037/a0021279

Reichle E D, Liversedge S P, Pollatsek A, Rayner K. 2009. Encoding multiple words simultaneously in reading is implausible. Trend in Cognitive Sciences 13: 115-119 DOI 10.1016/j.tics.2008.12.002

Reichle E D, Rayner K, \& Pollatsek A. 2003. The E-Z reader model of eye-movement control in reading: comparisons to other models. Behavioral and Brain Sciences, 26: 445-526, DOI 10.1017/S0140525X03000104 •

Salthouse T A. 1994. The nature of the influence of speed on adult age differences in cognition. Developmental Psychology 30: 240-259 DOI org/10.1037/0012-1649.30.2.240

Shen D, Liversedge S P, Tian J, Zang C, Cui L, Bai X, Yan G, Rayner K. 2012. Eye movements of second language learners when reading spaced and unspaced Chinese text. Journal of Experimental Psychology: Applied 18: 192-202 DOI $10.1037 / \mathrm{a} 0027485$.

Shen W, Li X. 2012. The uniqueness of word superiority effect in Chinese reading (in Chinese). Chinese Science Bulletin (Chinese Version) 57: 3414-3420 DOI 10.1360/972012-666

Shu H, Zhou W, Yan M, \& Kliegl R. 2011. Font size modulates saccade-target selection in Chinese reading. Attention, Perception, \& Psychophysics, 73, 482- 490 DOI 10.3758/s13414-010-0029-y

Stine-Morrow E A L, Miller L M S, Herzog C. 2006. Aging and self-regulated language processing. Psychological Bulletin, 132: 582-606 DOI 10.1037/0033-2909.132.4.582

Venables W N, Ripley B D. 2002. Modern applied statistics with S (4th ed.). New York, NY: Springer.

Wang J, Li L, Li S, Xie F, ChangM, Paterson K B, White S J, \& McGowan V A. 2016. Adult age differences in eye movements during reading: The evidence from Chinese. Journals of Gerontology: Psychological Sciences, 71: DOI 
664

665

666

667

668

669

670

671

672

673

674

675

676 10.1093/geronb/gbw036.

Yan M, Kliegl R, Richter E M, Nuthmann A, \& Shu H. 2010. Flexible saccade-target selection in Chinese reading. The Quarterly Journal of Experimental Psychology, 63,705-725 DOI 10.1080/17470210903114858

Yan M, Zhou W, Shu H, Kliegl R. 2015. Perceptual span depends on font size during the reading of Chinese sentences. Journal of Experimental Psychology: Learning, Memory, and Cognition 41: 209-219 DOI 10.1037/a0038097

Zang C, Zhang M, Bai X, Yan G, Paterson K B, \& Liversedge S P. 2016. Effects of word frequency and visual complexity on eye movements of young and older Chinese readers. The Quarterly Journal of Experimental Psychology, 69: 1409-1425 DOI 10.1080/17470218.2015. 1083594

Zhang X X, Fang Z, Du Y C, Kong L. Y, Zhang Q, Xing Q. 2012. The centro-parietal N200: An event-related potential component specific to Chinese visual word recognition. Chinese Science Bulletin 57: 1516-1532 DOI 10.1007/s11434-0114932-y

Zhou X L, Marslen-Wilson W. 2000. The relative time course of semantic and phonological activation in reading Chinese. Journal of Experimental Psychology: Learning, Memory, and Cognition 26: 1245-1265 DOI 10.1037//0278-7393.26.5.1245 


\section{Figure 1 (on next page)}

Figure 1 Examples of the word $\mathrm{n}$ disappearance conditions

(note: the asterisk indicates a fixation location). 


\begin{tabular}{|c|c|c|}
\hline (a) & $\begin{array}{l}\text { 垃圾通道需要及时清理。 } \\
*\end{array}$ & [beginning of fixation] \\
\hline (b) & $*$ 通道需要及时清理。 & {$[\mathrm{after} 40 / 80 / 120 / 160 \mathrm{~ms}]$} \\
\hline (c) & $\begin{array}{c}\text { 垃圾通道需要及时清理。 } \\
*\end{array}$ & [a new fixation] \\
\hline (d) & 垃圾 ${ }_{*}$ 需要及时清理。 & [after40/80/120/160ms] \\
\hline (e) & $\begin{array}{c}\text { 垃圾 需要及时清理。 } \\
*\end{array}$ & [An immediate re-fixation did't result word reappearance] \\
\hline
\end{tabular}




\section{Figure 2 (on next page)}

Figure 2 Examples of the four word $n+1$ disappearance conditions

(note: the asterisk indicates a fixation location) 


\begin{tabular}{|c|c|c|}
\hline (a) & $\begin{array}{l}\text { 垃圾通道需要及时清理。 } \\
\text { ． }\end{array}$ & [beginning of fixation] \\
\hline (b) & $\begin{array}{c}\text { 垃圾 需要及时清理。 } \\
*\end{array}$ & [after40/80/120/160ms] \\
\hline (c) & $\begin{array}{c}\text { 垃圾通道需要及时清理。 } \\
*\end{array}$ & [a new fixation] \\
\hline (d) & $\begin{array}{c}\text { 垃圾通道 } \\
*\end{array}$ & [after40/80/120/160ms] \\
\hline (e) & $\frac{\text { 垃圾通道 }}{*}$ & [An immediate re-fixation did't result word reappearance] \\
\hline
\end{tabular}




\section{Figure 3 (on next page)}

Figure 3. Sentences reading time of young and old adults for each of the five presentation conditions in two experiments

Error bars show the standard error each group under each condition. In each group of the bars, the first bar was the data of old adults when reading word $\mathrm{n}$ disappearing text, the second was the data of young adults when reading word $n$ disappearing text, the third was the data of old adults when reading word $n+1$ disappearing text, and the fourth was the data of young adults when reading word $n+1$ disappearing text 


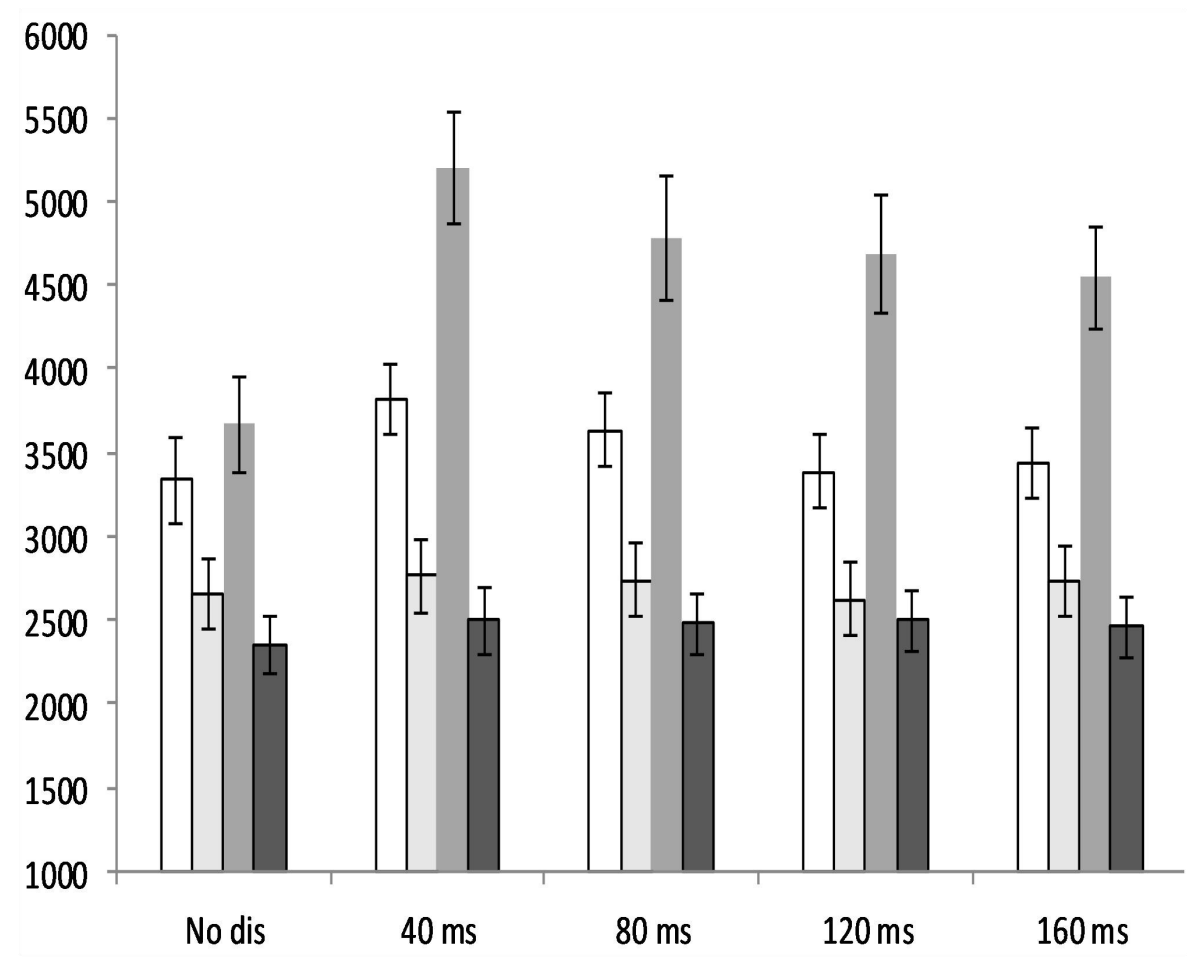




\section{Figure 4 (on next page)}

Figure 4. Mean gaze duration of young and old adults for each of the five presentation conditions in the two experiments

(conventions as for Figure 3) 


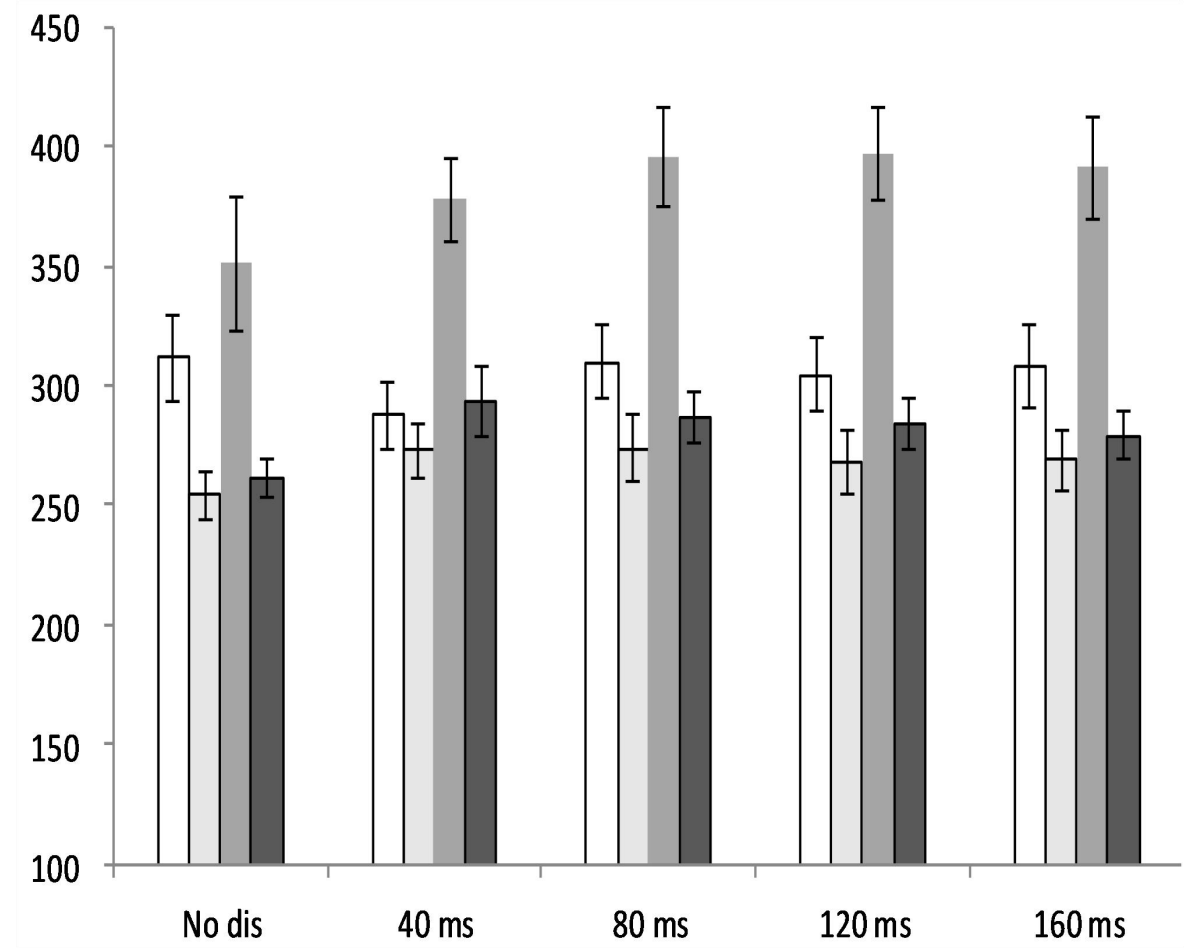




\section{Table $\mathbf{1}$ (on next page)}

Table 1 The mean and standard deviations of the measures across conditions and age groups in Experiment 1

Means and standard deviations are computed across subjects' means. The standard deviations are given in parentheses. Control, the normal display condition; RT, sentence reading time in millisecond; MeanGazeDur, mean gaze duration in milliseconds; Pro.Sikp, probability of words skipped in the initial pass reading; NO.Reg, number of regressions. 


\begin{tabular}{cccccc}
\hline & & RT & MeanGazeDur & Pro.Skip(\%) & NO.Reg \\
\hline \multirow{3}{*}{ Young } & Control & $2654(816)$ & $254(38)$ & $24.9(15.4)$ & $1.7(1.1)$ \\
adults & $40 \mathrm{~ms}$ & $2761(822)$ & $273(46)$ & $25.3(15.6)$ & $2.1(1.1)$ \\
& $80 \mathrm{~ms}$ & $2738(848)$ & $274(56)$ & $25.0(15.1)$ & $1.9(0.9)$ \\
& $120 \mathrm{~ms}$ & $2625(840)$ & $268(51)$ & $25.5(16.9)$ & $1.8(1.0)$ \\
& $160 \mathrm{~ms}$ & $2728(813)$ & $269(49)$ & $25.5(17.3)$ & $1.7(1.0)$ \\
\multirow{3}{*}{ Aged } & Control & $3334(1001)$ & $312(71)$ & $13.5(8.3)$ & $2.1(0.9)$ \\
adults & $40 \mathrm{~ms}$ & $3815(819)$ & $288(55)$ & $18.1(8.8)$ & $3.5(0.9)$ \\
& $80 \mathrm{~ms}$ & $3632(835)$ & $310(61)$ & $16.5(10.1)$ & $3.0(1.0)$ \\
& $120 \mathrm{~ms}$ & $3389(816)$ & $304(60)$ & $15.4(10.2)$ & $2.7(1.0)$ \\
& $160 \mathrm{~ms}$ & $3439(835)$ & $308(68)$ & $16.6(9.4)$ & $2.6(1.0)$
\end{tabular}

1 


\section{Table 2 (on next page)}

Table 2 The mean and standard deviations of the measures across conditions and age groups in Experiment 2

Means and standard deviations are computed across subjects' means. The standard deviations are given in parentheses. Control, the normal display condition; RT, sentence reading time in millisecond; MeanGazeDur, mean gaze duration in milliseconds; Pro.Sikp, probability of words skipped in the initial pass reading; NO.Reg, number of regressions. 


\begin{tabular}{cccccc}
\hline & & RT & MeanGazeDur & Pro.Skip(\%) & NO.Reg \\
\hline \multirow{3}{*}{ Young } & Control & $2347(640)$ & $262(31)$ & $21.1(9.4)$ & $1.4(0.7)$ \\
adults & $40 \mathrm{~ms}$ & $2498(762)$ & $293(57)$ & $26.8(9.4)$ & $1.5(0.8)$ \\
& $80 \mathrm{~ms}$ & $2475(723)$ & $287(42)$ & $26.8(9.2)$ & $1.6(0.8)$ \\
& $120 \mathrm{~ms}$ & $2494(691)$ & $284(42)$ & $24.8(8.7)$ & $1.5(0.8)$ \\
& $160 \mathrm{~ms}$ & $2460(700)$ & $279(39)$ & $24.7(8.5)$ & $1.5(0.8)$ \\
Aged & Control & $3669(1123)$ & $351(108)$ & $7.6(4.6)$ & $1.9(0.9)$ \\
adults & $40 \mathrm{~ms}$ & $5195(1299)$ & $378(67)$ & $14.3(4.7)$ & $4.4(2.1)$ \\
& $120 \mathrm{~ms}$ & $4784(1440)$ & $396(80)$ & $14.0(6.0)$ & $3.6(1.7)$ \\
& $160 \mathrm{~ms}$ & $4687(1331)$ & $396(75)$ & $13.0(6.5)$ & $3.3(1.6)$ \\
\hline
\end{tabular}

1

2 